\title{
USO Y MODIFICACIÓN DE LAS NOTAS DE PRENSA DEPENDIENDO DE LAS CARACTERÍSTICAS DE LOS PERIÓDICOS
}

Lorena Busto- Salinas': Universidad de Burgos. España lbusto@ubu.es

\section{RESUMEN}

La nota de prensa es una de las herramientas más importantes y habituales de los gabinetes de prensa para transmitir una notica a los medios de comunicación. Se trata de un texto informativo breve sobre un tema de actualidad de la entidad que lo emite y que suele escribirse de la misma manera en que lo haría un redactor para que sufra la menor modificación posible. Los periódicos utilizan estos documentos para llenar sin demasiada complejidad sus páginas, aunque como cada uno de ellos posee sus propias características, parece razonable pensar que usan estos textos de manera diferente. Para comprobar esta hipótesis, se han estudiado las notas de prensa enviadas durante cuatro años por la Fundación Atapuerca (Burgos, España) y se ha analizado su repercusión en seis periódicos de diferente tirada y ámbito geográfico. Entre los resultados más relevantes, cabe señalar que los diarios locales publican notablemente más notas de prensa y de cualquier temática; además, suelen incluirlas más a menudo en portada. Sin embargo, se han detectado algunos aspectos que no sufren demasiadas modificaciones entre unos periódicos y otros, como, por ejemplo, el tamaño de la información, el grado de reelaboración o la inclusión de fotografías.

PALABRAS CLAVE: Relaciones públicas - Comunicación institucional - Análisis de prensa - Nota de prensa

\footnotetext{
${ }^{1}$ Autor Correspondiente

Lorena Busto- Salinas: Investigadora FPI. Facultad de Humanidades y Educación. Universidad de Burgos, España.

Correo: 1 lbusto@ubu.es
} 


\title{
USE AND MODIFICATION OF PRESS RELEASES DEPENDING ON THE TYPE OF NEWSPAPER
}

\begin{abstract}
The press release is one of the most important tools used by press offices to transmit a story to the mass media. It consists on a short text regarding an organization's newsworthy story written in the same way as a journalist would write it. The purpose of a press release is to be published by the mass media with the less modification as possible. These documents are used by newspapers to easily fill their pages with news, although they might modify its lines differently depending on the type of daily. In order to prove this hypothesis, all press releases sent in four years to the mass media by the Atapuerca Foundation (Burgos, Spain) have been studied, as well as their impact in six different newspapers. One of the most relevant results is that local newspapers publish more news releases and of any kind of subject, placing them more often in front page than their counterparts. Nevertheless, it has also been noticed some aspects of the press release that doesn't change much between some newspapers and others, like the size of the information, the degree of reelaboration or the usage of images and their dimensions.
\end{abstract}

KEY WORDS: Public relations - institutional communication - press analysis - press release

\section{INTRODUCCIÓN}

No existe en la actualidad ninguna investigación que detalle el diferente tratamiento que sufren las notas de prensa según las características de los periódicos que las reciben. Los académicos, hasta ahora, se han limitado a analizar la publicación o no de una nota de prensa según la tirada del periódico, pero han dejado a un lado otros aspectos relevantes de la divulgación, como el tamaño del texto, la aparición en portada, la utilización de información de archivo, la inclusión de fotografías, etc.

Además, bien por desconocimiento o por falta de recursos, muchos gabinetes de comunicación continúan enviando notas de prensa a los medios de comunicación de manera masiva, sin personalizar cada texto según el diario al que se dirige. De esta manera, un pequeño periódico de una reducida ciudad recibe el mismo texto que un gran diario especializado, cuando las posibilidades de cada uno de ellos son, a priori, desiguales. Los diarios grandes suelen tener más redactores especializados y gozan de más fuentes de información; los pequeños, por el contrario, cuentan con menos periodistas y no pueden profundizan tan ampliamente las noticias. 
La bibliografía existente acerca de la publicación o no de las notas de prensa según las características de cada periódico, a pesar de no ser muy numerosas teniendo en cuenta la cantidad total de indagaciones que existe en torno a esta herramienta, ya atisba cierta discrepancia entre unos diarios y otros.

Por ejemplo, en la década de los 70, Drew y Wilhoit (Drew \& Wilhoit, 1976) apuntaban que los periódicos de pequeña tirada favorecen las informaciones locales, mientras que los que tienen una amplia difusión prefieren publicar todo tipo de temas. Stone, Gerald y Morrison (Stone, Gerald \& Morrison, 1976) también constatan diferencias en el tipo de contenidos que publican. Según indican, los periódicos de gran tirada contienen más artículos de pago, mientras que los pequeños incluyen más contenidos de sociedad, crónicas y anuncios legales. Debido a su reducida difusión, gozan de menos publicidad nacional, menos fotografías locales y un mayor porcentaje de noticias procedentes de relaciones públicas. Lacy y Bernstein (Lacy \& Bernstein, 1988) comparten estos argumentos, e indican que los periódicos de pequeña circulación reservan más espacio que los de mayor tirada a las noticias procedentes de fuentes externas, como los comunicados de los gabinetes de comunicación.

La idea de que los periódicos pequeños utilizan más notas de prensa que sus competidores más grandes ha sido analizada en varias investigaciones por Lisa Morton. Así, llega a la conclusión de que, a medida que la circulación aumenta, las posibilidades de que un comunicado se publique disminuyen (Morton, 1984), algo que corrobora también junto a John Warren (Warren, 1992). En este último estudio establecen que los diarios de más de 20.000 ejemplares divulgan significativamente menos notas de prensa. Debido a la escasa plantilla de redactores, los periódicos pequeños son mucho más susceptibles de publicar aquellas notas de prensa que estén "listas para publicar", es decir, aquellas que cumplan con los parámetros de la redacción informativa (Morton, 1988).

A pesar de que Morton y Ramsey (Morton \& Ramsey 1994) no han hallado diferencias significativas entre el tipo de fuente emisora y su posterior publicación en la prensa - independientemente del tipo de diario-, algunos expertos insisten en que hay aspectos de una organización que pueden ayudar a mejorar el número de informaciones publicadas. Uno de ellos es el nivel humano y profesional de las personas encargadas de la comunicación de una empresa. Según sostienen, establecer una relación personal entre el profesional del gabinete de prensa y el periodista suscita un mayor nivel de publicaciones (Walters \& Walters, 1992) y ayuda a dibujar en la mente del redactor una fuente de información ética y hábil (Jeffers, 1977).

Por otra parte, también es relevante la inclusión de nombres propios, tanto de la institución o empresa como de alguno de sus representantes, ya que incrementa la posibilidad de que los periodistas utilicen estas referencias (Sanford, Moar \& Garrod, 1988). Estos sujetos, además, pueden pasar a constituir la principal fuente de referencias futuras sobre una temática concreta (Downing, 1996). 
La proximidad física de la institución respecto a la sede del medio de comunicación también afecta a las decisiones del redactor (Aronoff, 1976; Whitlow, 1977), lo que podría propiciar una mayor inserción en los periódicos locales que en los nacionales. De hecho, algunos autores han querido ahondar más allá y han analizado si son los organismos los que verdaderamente hacen que una nota de prensa tenga más opciones de publicación que otra y no el tipo de periódico. Diversas investigaciones sustentan la idea de que las fuentes de información estables, creíbles y fiables obtienen una mayor cobertura mediática (Fishman, 1980); (Gitlin, 1980); (Goodell, 1975); (Tuchman, 1978); (Wilkie, 1996). También aquellas que se muestran como fidedignas y autorizadas (Gans, 2004), que han creado un clima de confianza (Morton, 1986) y que cuentan en su historial con informaciones de calidad (Dunwoody \& Ryan, 1983); (Stocking, 1985) son más propensas a aparecer en los periódicos. Estos calificativos se consiguen conforme se asienta un gabinete de prensa con el paso del tiempo, tal y como se aprecia en un estudio de caso sobre los comunicados de una entidad y su repercusión en prensa. A pesar de que los documentos despachados no sufren un aumento progresivo en los años analizados, su utilización por parte de los diarios crece cada ejercicio (Busto, 2011).

\subsection{Justificación y objetivos}

A pesar de que existen numerosas investigaciones acerca de la nota de prensa, la mayoría proceden del ámbito anglosajón, dejando la producción española como un elemento casi residual. Como tal, no está claro si las conclusiones que proporcionan esos estudios se pueden extrapolar directamente a España. Por otra parte, muchas de las indagaciones llevadas a cabo desde el nacimiento de esta herramienta se realizaron en las décadas de 1960 a 1990, dejando el siglo XXI sin apenas producción científica al respecto, un hecho contradictorio teniendo en cuenta que el uso de las notas de prensa ha aumentado en los últimos años. Con todo, no se tiene constancia de si los resultados que hace unas décadas parecían inalterables lo siguen siendo hoy en día.

Además, muy pocas estudiosos han indagado acerca de la repercusión en prensa más allá de su mera publicación. Esto significa que los investigadores no han adentrado en otros aspectos importantes de la divulgación, como el tamaño del texto, la inclusión de fotografías, la aparición en portada, la utilización de información de archivo, etc.

Por todo ello, se presenta esta investigación, cuyo principal objetivo es determinar si hay alguna diferencia entre los periódicos de diferente ámbito geográfico a la hora de publicar las notas de prensa. Asimismo, se pretende averiguar si existe alguna diferencia en el tratamiento de la noticia; es decir, si algunos periódicos son más propensos a incluir las notas con un tamaño más extenso, en primera página, acompañadas de fotografía, con una mayor elaboración propia del redactor, etc. 


\section{METODOLOGÍA}

Con el fin de conocer las diferencias en el tratamiento de una nota de prensa según el ámbito geográfico de los periódicos, se han estudiado todos los comunicados enviados a los medios de comunicación desde 2007 a 2010 por la Fundación Atapuerca. Esta entidad tiene como misión apoyar y difundir los yacimientos del mismo nombre, situados en Burgos, y declarados Patrimonio de la Humanidad en 2000. No inserta publicidad en ningún medio de comunicación, por lo que se trata de una organización perfecta para analizar su comunicación exterior sin temor a que los resultados puedan ser alterados por la compra de espacios. En total, se han recogido 164 notas de prensa.

Por otro lado, se ha analizado la repercusión de estas notas de prensa en seis periódicos diarios de diferentes ámbitos geográficos. Estos son dos localesprovinciales: Diario de Burgos y El Correo de Burgos; dos regionales: El Norte de Castilla y $\mathrm{ABC}$ en su versión de Castilla y León; y dos nacionales: El País y La Vanguardia. Se han encontrado 240 textos periodísticos provenientes de los comunicados de la Fundación Atapuerca.

Finalmente, se ha utilizado un programa estadístico para establecer relaciones entre algunos aspectos de la nota de prensa y su repercusión según la tirada de los periódicos. En algunos casos, además, se han realizado pruebas estadísticas para cerciorarse de que los resultados son estadísticamente fiables, es decir, que no son fruto de la casualidad. La significación se ha establecido en igual o menor de 0,005, de tal forma que las relaciones sean claramente significativas y no valga cualquier pequeña diferencia entre lo esperado y lo encontrado.

\section{ANÁLISIS Y DISCUSIÓN}

La primera discrepancia entre unos periódicos y otros se halla, mismamente, en el número de notas de prensa publicadas. Los locales, por ejemplo, son los diarios que más textos publican. De los 164 documentos enviados por la Fundación Atapuerca, ellos divulgan 115, dando un índice de penetrabilidad del 70,1\%. Después de ellos están los regionales, que utilizan 35 de esos comunicados. El porcentaje de uso en este caso baja considerablemente respecto a los locales y se sitúa en el $21,3 \%$. Los nacionales, con 3 notas de prensa publicadas, arrojan una cifra porcentual mínima: el $1,8 \%$.

Como algunas notas de prensa han sido divulgadas por más de un diario a la vez, la suma total de textos periodísticos que ha visto la luz asciende a 240 , una cifra que dividida en bloques se corresponden con: 187 en los locales, 48 en los regionales y 5 en los nacionales. 


\subsection{Sección}

Los periódicos locales incluyen la mayoría de las informaciones sobre las notas de prensa de la Fundación Atapuerca en la sección "Burgos". En el caso de Diario de Burgos, ocupa el 66,7\% del total, y en El Correo de Burgos, el 78\%. La segunda sección más recurrente en ambos casos se queda bastante alejada de la primera. En Diario de Burgos, se trata de "Vivir", que reúne el 21,9\% de los casos; la sección "Provincia" de El Correo de Burgos se queda con el 9,8\%.

En los periódicos regionales, sin embargo, no existe una diferencia tan acusada como en los locales, aunque hay secciones que prevalecen por encima de otras. Por ejemplo, la más recurrente en El Norte de Castilla es la de "Cultura", con 12 apariciones, aunque la sigue de cerca "Vida \& Ocio", que suma 8. En porcentajes se corresponde con el 52,2\% y el 34,8\%, respectivamente. En ABC publican la mayor parte de los textos de la Fundación (el 66,7\%) en "Castilla y León", mientras que la segunda sección con más informaciones, "Sociedad", se queda en el 25\%. El resto de secciones de ambos diarios no llegan por sí solas a sumar el 10\% del total.

Los periódicos nacionales, por su parte, únicamente incluyen las noticias de la Fundación Atapuerca en una sola sección. El País lo hace en la de "Sociedad" y La Vanguardia, en "Tendencias".

\subsection{Número de página}

Un mecanismo simple para comprobar la importancia que le otorga un periódico a una noticia es observar si se incluye en página par o impar. Las que aparecen en una cara impar obtienen, a priori, una mayor relevancia, puesto que son más susceptibles de ser leídas por los lectores antes que las pares. En la muestra analizada, existen pequeñas diferencias en cada periódico en particular, pero no puede decirse que sea debido al factor de distribución o a la proximidad física. Diario de Burgos publica más textos en la página par (65,7\% frente al 34,3\% de las impares), al igual que El Correo de Burgos (52,4\% frente a 47,6\%) y El Norte de Castilla $(65,2 \%$ frente a 34,8\%).

En el otro lado de la balanza se encuentran ABC y La Vanguardia, que acumulan unas cifras porcentuales de páginas impar del 56\% y 66,7\%, respectivamente. Por su parte, El País ha publicado las dos informaciones procedentes de la Fundación Atapuerca en página par.

\subsection{Autores}

Existe un gran número de autores que firman en los periódicos las informaciones procedentes de las notas de prensa de la Fundación Atapuerca, y son los locales los que arrojan una cifra más alta. En Diario de Burgos, por ejemplo, existen 27 firmas diferentes, y en El Correo de Burgos, 17. Por detrás de ellos, se encuentran los regionales: El Norte de Castilla, con 9, y ABC, con 6. En cuanto a los nacionales, son los que menos variedad presentan, ya que tanto El País como La Vanguardia poseen 
Otra diferencia acerca de los autores de las informaciones es que los que escriben en diarios nacionales están especializados en periodismo científico. Estos son Alicia Rivera y Mónica Salomé, ambas de El País, y Josep Corbella, de La Vanguardia (la otra firma de este periódico es la agencia de noticias Efe). En los regionales, son normalmente los corresponsales en la provincia los que cubren las noticias sobre Atapuerca: Mar González, en ABC, y Rosalía Santaolalla, en El Norte de Castilla. En el caso de los locales, ningún redactor de los que firman las noticias de la Fundación Atapuerca tiene formación en periodismo científico. Pese a ello, en Diario de Burgos, hay una periodista (Carmen Mendieta) que suele escribir gran parte de los textos. Sin embargo, en El Correo de Burgos no parece haber un redactor encargado de las noticias de la Fundación Atapuerca, ya que la persona con más contribuciones, Marta Casado, firma únicamente 8 textos $(9,8 \%)$.

\subsection{Tema}

También existen diferencias en la publicación de unas notas de prensa en unos diarios y otros según el tema que se trate. Para comprobar este dato, se han dividido las noticias de la Fundación Atapuerca en: comunicados institucionales, acuerdos de colaboración, actividades de divulgación, investigación, yacimientos, premios y otros. Se ha de apreciar que la investigación y los yacimientos son los asuntos más propiamente característicos de esta fundación; es decir, se trata de temas peculiares cuyas características no pueden proporcionar fácilmente otras entidades (al contrario de lo que sucede con los comunicados institucionales, los acuerdos de colaboración o los premios, por ejemplo).

Con esta división, se puede observar que los periódicos locales publican más notas de prensa sobre los yacimientos, la investigación, las colaboraciones y los comunicados institucionales, en este orden. Los porcentajes de penetración en cada uno de estos asuntos son, respectivamente: 92,3\%, 87,5\%, 84,6\% y 81,0\%. Los regionales, en cambio, prestan mucha más atención a las notas sobre investigación que a cualquier otro tema. El $75 \%$ de estos textos fueron cubiertos por este par de periódicos, cuando el segundo asunto más recurrente, los yacimientos, no llega al $40 \%$. Por su parte, lo nacionales únicamente han publicado noticias sobre investigación. Tres de las ocho notas enviadas (el 37,5\%) han aparecido en por lo menos un diario nacional.

De hecho, realizando una prueba estadística de Chi-cuadrado, se puede observar que existe una relación significativa entre el tema investigación y los diarios regionales y nacionales. Esto significa que la relación es estadísticamente fiable y no fruto del azar. Seis de los ocho comunicados que trataban estos asuntos fueron publicados por los diarios regionales (75\%). Cuando se trata de otra temática, el nivel de penetración en este grupo cae hasta el 18,6\%. En cuanto a los nacionales, las cinco informaciones que publicaron El País y La Vanguardia en los cuatro años analizados provienen exclusivamente de documentos de esta temática; del resto, no hicieron uso de ninguno. 
Con todos estos datos, se puede establecer que los periódicos que abarcan un amplio espectro prestan más atención a aquellas notas de prensa que exponen un tema que se distancia de lo que otras fuentes informativas ofrecen y que se muestra como una información exclusiva que no puede adquirirse por otros procedimientos. Aquellos periódicos cuyo ámbito geográfico es más reducido, no prestan tanta atención a este aspecto y prefieren publicar informaciones de todo tipo.

\subsection{Portada}

A medida que aumenta la difusión de un periódico, es más difícil que una nota de prensa aparezca en portada. En los diarios nacionales analizados en este estudio, por ejemplo, no se ha encontrado ninguna referencia en primera página. Los regionales, en cambio, publican 4 , y los locales, 54 . Si se comparan estos datos con el conjunto de notas publicadas por cada grupo, se observa que el $28,8 \%$ de las noticias utilizadas por los locales tienen una mención en primera página. En los regionales, la cifra es mucho más baja: el 8,3\%. También se aprecian diferencias en el tema de las notas de prensa que suelen aparecer en esa página. Tres de las cuatro portadas de los periódicos regionales versan sobre investigación; la restante trata sobre los yacimientos. En cambio, los locales hacen más referencias en primera página a los acuerdos de colaboración (con 14), a los yacimientos (11) y a la investigación y los comunicados institucionales (9 cada uno).

\subsection{Tamaño del texto}

No hay demasiadas diferencias en el tamaño que suelen ocupar los textos periodísticos procedentes de las notas de prensa de la Fundación Atapuerca. La media de módulos de las informaciones en todos los diarios se encuentran en la veintena, a excepción de El País, cuya media es de 30 (para el cálculo de módulos se toma como referencia la publicidad en el diario de información general de más tirada de España, El País, que concibe 40 en cada página). El segundo periódico con una mayor media es La Vanguardia, con 26, seguido de Diario de Burgos, con 23,70. El Correo de Burgos, por su parte, suele publicar noticias de unos 22,98 módulos, y $\mathrm{ABC}$, de 22,16. La media más baja se halla en El Norte de Castilla, que apenas sobrepasa las 20 divisiones (más concretamente 20,83). Tampoco existen diferencias notables en el número medio de columnas (4,17 en los locales, 3,93 en los regionales y 4,41 en los nacionales).

\subsection{Fotografía}

En la mayoría de los casos, los periódicos incluyen al menos una fotografía en las informaciones provenientes de las notas de prensa de la Fundación Atapuerca. Los locales lo hacen en el 76,03\% de las noticias publicadas, los regionales, en el 75,11\%, y los nacionales, en el 83,33\%. 
Además, el tamaño medio de las imágenes se mantiene bastante similar de unos a otros. Tomando como referencia los 40 módulos por página que calcula El País, se aprecia que los locales suelen insertar fotografías de unos 9,78 módulos, los regionales de 10,18 y los nacionales de 9,25. Como se ve, las imágenes de los diarios regionales son algo más grandes, pero la diferencia es ínfima.

\subsection{Rueda de prensa}

Algunas de las notas de prensa que envió la Fundación Atapuerca durante cuatro años estaban vinculadas a algún encuentro con los medios. En concreto, 73 de los 164 documentos despachados. Con el fin de comprobar si existe una relación significativa entre la convocatoria de una rueda de prensa y la posterior publicación de la noticia, se ha realizado una prueba de Chi-cuadrado. En sus resultados se ha podido comprobar que sí que existe una dependencia entre ambas variables, pero únicamente en los diarios locales. De las 73 ruedas de prensa que convoca la Fundación Atapuerca, Diario de Burgos anuncia 64 (el 87,7\%); cuando no existe una convocatoria, el grado de publicación baja al 45,1\%. En El Correo de Burgos ocurre algo similar. Publica 55 de esos eventos, alcanzando el 75,3\%. Cuando no se celebra ninguno, el porcentaje de publicación baja al 29,7\%. Por el contrario, los diarios regionales y nacionales no muestran el mismo grado de dependencia, por lo que parece que no prestan demasiada atención a una reunión con los medios.

También se ha querido comprobar si la convocatoria de una rueda de prensa repercute en el tratamiento de la noticia en prensa, por ejemplo, a través del tamaño que se le otorga. Para ello, se ha realizado una prueba $\mathrm{T}$ para dos muestras independientes. En las variables para contrastar se han introducido los módulos de las informaciones, y en la variable de agrupación, la convocatoria o no a los medios. En ningún caso analizado -ni en los periódicos por separado, ni por ámbito geográfico ni en conjunto- se han hallado diferencias significativas. Se puede afirmar, entonces, que una rueda de prensa no influye estadísticamente en el tamaño medio de las informaciones independientemente de las características del periódico.

\subsection{Personajes nombrados}

Algunos manuales de relaciones públicas instan a incorporar alguna declaración o una simple mención de algún representante relevante de una organización en las notas de prensa, de tal manera que se pueda observar el aspecto humano de la noticia. En el caso de la Fundación Atapuerca, 113, es decir, el 68,9\%, hacían referencia a alguna persona. Para averiguar si este truco tiene realmente algún efecto en los periodistas que lo reciben, se han anotado y diferenciado las personas que aparecen en los documentos de esta investigación según la siguiente lista: Juan Luis Arsuaga, José María Bermúdez de Castro, Eudald Carbonell (ellos tres codirectores de las excavaciones), Emiliano Aguirre (considerado el "padre" de los yacimientos) y otros miembros de la Fundación Atapuerca. El resto de personas, debido a la variedad y al alto número de apariciones, se han englobado en protagonistas científicos, institucionales, empresariales v culturales. 
Realizando una prueba de Chi-cuadrado, se aprecia que la aparición de al menos una persona en las notas de prensa no asegura que el texto se vaya a publicar en ninguna clase de periódico. Tampoco existe una relación significativa entre publicación y la inclusión en el texto de al menos un codirector.

Sin embargo, separando las referencias según personas o grupos se encuentran varias coincidencias. Por ejemplo, que existen mayores probabilidades de que los diarios regionales publiquen una noticia en la que se nombra a algún personaje institucional. Cuando se incluye una mención, las posibilidades de utilización de la nota de prensa se sitúan en el 45,8\%, mientras que cuando no existe esta referencia, el porcentaje baja hasta el $17,1 \%$.

Ocurre justamente lo contrario con los periódicos locales y los protagonistas científicos. Cuando en una nota de prensa se menciona a este tipo de personas, las probabilidades de publicación bajan del 75,19\% al 48,39\%. La significación en 0,003 muestra que se trata de una relación significativa.

En cuanto al tratamiento informativo que le dan los periódicos a las notas de la Fundación Atapuerca, se ha encontrado una relación significativa. Utilizando la prueba $\mathrm{T}$ para dos muestras independientes se puede observar que cuando los tres codirectores de las excavaciones son nombrados en una misma nota de prensa, los periódicos regionales publican una información media de 35,21 módulos; cuando no se incluye a los codirectores en conjunto, la extensión baja hasta los 15,11 módulos. Hay que concretar, pese a todo, que cuando intervienen estos personajes juntos es porque se trata de un asunto de la máxima importancia, por lo que resulta normal que los medios publiquen esas noticias más extensas. En el resto de casos, no se han encontrado diferencias significativas, aunque todos los diarios componen una noticia más amplia cuando se nombran a estas tres personas.

\subsection{Elaboración propia}

A pesar de que en un principio puede parecer que los periódicos nacionales, al poseer una plantilla de redactores especializados en cada temática, agregan una mayor modificación a las noticias, las cifras no sustentan esta idea. Se ha calculado el porcentaje de elaboración propia que introducen los periodistas en las noticias de la Fundación Atapuerca; es decir, la proporción de datos y frases que no aparecían previamente en las notas de prensa. Tanto los locales, como los regionales y los nacionales arrojan una media de elaboración propia por noticia muy similar: en el caso de los locales se mantiene en el $60,64 \%$, en los regionales sube hasta el $65,40 \%$ y en los nacionales se queda en un $60,59 \%$.

En cualquier caso, en su mayoría, los textos que aparecen en los periódicos analizados sobre las notas de prensa de la Fundación Atapuerca estaban o bien acompañados de mucha elaboración propia del periodista (de un 80 a un $100 \%$ ) o bien de muy poca (de un 0 a un $20 \%$ ). 


\subsection{Archivo}

Se ha querido comprobar si los periódicos con mayor difusión insertan más datos de archivo a las noticias que reciben o si, por el contrario, no tienden a contextualizar las informaciones tanto como los pequeños. Según los datos recogidos en esta investigación, se puede determinar que, al menos para las notas de prensa de la Fundación Atapuerca, cuanto más grande es la difusión de un periódico, más datos de archivo se suele incluir en las noticias.

En la muestra analizada en este estudio, los nacionales incluyeron información propia de archivo en el $66,67 \%$ de las informaciones.

Los regionales, por su parte, insertan este tipo de información en el 31,4\% de los casos y los locales, en el $21,9 \%$.

\section{CONCLUSIONES}

La nota de prensa es un recurso clave para un gabinete de comunicación a la hora de transmitir una noticia a la prensa. Sin embargo, no todos los periódicos poseen las mismas características y, parece lógico pensar, no tratan los documentos que reciben de manera idéntica. En esta investigación, se ha llevado a cabo un estudio de caso para detectar qué notas se suelen publicar más y con qué tratamiento informativo según la tirada y ámbito geográfico de los periódicos.

Así, se observa que cuanto más pequeño es el ámbito de difusión de un diario, mayores posibilidades existen de que una nota de prensa sea divulgada. En el caso de la Fundación Atapuerca, por ejemplo, el porcentaje de publicación en los locales, regionales y nacionales es del 70,1\%, 21,3\% y 1,8\%, en este orden.

Además, cuando un diario local da a conocer la información de una nota de prensa, suele incluirla más frecuentemente en primera página. En la investigación desarrollada en este artículo se ha comprobado que el $28,8 \%$ de las noticias publicadas aparecen en portada en los diarios locales, mientras que los regionales lo hacen en el $8,3 \%$. Los nacionales no divulgan ninguna noticia proveniente de la Fundación Atapuerca en esta página.

Por otra parte, los periódicos pequeños tienden a publicar casi cualquier tipo de documento, independientemente del tema principal, mientras que los más grandes prestan atención únicamente al asunto más característico de una entidad (en el caso de la Fundación Atapuerca, la investigación). Eso sí, a medida que aumenta la tirada, los redactores suelen incluir más información propia de archivo.

En cuanto a la autoría de los textos informativos, conviene señalar que los redactores que suelen escribir las noticias de la Fundación Atapuerca en los diarios nacionales están especializados en periodismo científico. 
En los regionales, entretanto, son normalmente los corresponsales los que cubren estas noticias. En uno de los locales, existe una periodista que suele informar sobre la Fundación Atapuerca, mientras en el otro local no parece haber un redactor clave.

En general, la mención de una persona en una nota de prensa no repercute en la posterior publicación de la noticia considerando como un único conjunto todos los periódicos analizados. Sin embargo, si se separan estos por ámbito geográfico y los personajes por categorías, se encuentran algunas diferencias significativas. Por ejemplo, los regionales publican más comunicados que mencionan a personajes institucionales y los locales utilizan menos notas con personas científicas.

En ocasiones, un gabinete de prensa envía a los medios de comunicación una nota de prensa que está vinculada a una rueda de prensa. Cuando esto ocurre en el caso de la Fundación Atapuerca, existen más posibilidades de que sea utilizada por algún periódico local, pero no sucede lo mismo con los regionales o nacionales. Tampoco existe ninguna diferencia clara en el tratamiento informativo que se le otorga a la noticia independientemente del tipo de diario y de su vinculación con un encuentro con los medios o no.

Aún con todo, existen algunos aspectos que no varían demasiado según el ámbito geográfico de los diarios, como, por ejemplo, el tamaño que ocupa la información. Tampoco se han detectado diferencias en el porcentaje de noticias que poseen una fotografía ilustrando el texto ni de la dimensión de estas imágenes. La tirada de un periódico tampoco parece estar relacionada con la elección de página par o impar a la hora de publicar un comunicado.

Otro elemento que no cambia de unos diarios a otros es la elaboración propia. Aunque parece comprensible pensar que los grandes periódicos -al poseer más periodistas y más especializados- reelaboran más profundamente las informaciones que llegan a sus mesas, esta investigación no sustenta esta idea, puesto que se ha observado que los diarios grandes introducen la misma elaboración propia que los pequeños a los comunicados de la Fundación Atapuerca.

\section{REFERENCIAS}

Aronoff, C. E. (1976). Predictors of success in placing releases in newspapers. Public Relations Review, 2: 43-57.

Busto, L. (2011). Eficacia de la nota de prensa en la comunicación institucional. El caso de la Fundación Atapuerca. Trabajo de investigación para la obtención de la Suficiencia Investigadora. Universidad de Burgos, Burgos.

Downing, P. A. (1996). Proper names as a referential option in English conversation en FOX, B. Amsterdam: Studies in anaphora John Benjamins. 
Drew, D. \& Wilhoit, C. G. (1976). Newshole allocation policies of American daily newspapers. Journalism Quarterly, 53: 434-440.

Dunwoody, S. \& Ryan, M. (1983). Public information persons as mediators between scientists and journalists. Journalism Quarterly, 59: 647-656.

EL PAÍS. Publicidad 2010. Recuperado el 16 de marzo de 2011, de: http:/ / www.elpais.com/ corporativos/ elpais/ publi.html?valor =3\&subvalor=1

Fishman, M. (1980). Manufacturing the news. Texas: University of Texas Press.

Gans, H. (2004). Deciding what's news: a study of CBS Evening News, NBC Nightly News, Newsweek and Time (24 ed.). Nueva York: Phanteon Books.

Gitlin, T. (1980). The whole world is watching: the mass media in the making and unmaking of the new left. California: University of California Press.

Goodell, R. (1975). The visible scientists. Boston: Little, Brown and Company.

Jeffers, D. W. (1977). Performance expectations as a measure of relative status of news and PR people. Journalism Quarterly, 54(2): 299-306.

Lacy, S. \& Bernstein, J. M. (1988). Daily newspaper content's relationship to publication cycle and circulation size. Newspaper Research Journal, 9: 49-57.

Morton, L. P. (1984). Tesis "The relationship of newspaper characteristics and types of releases on publications". Oklahoma State University. Oklahoma.

Morton, L. P. (1986). How newspapers choose the releases they use. Public Relations Review, 12(3): 22-27.

Morton, L. P. (1988). Effectiveness of camera-ready copy in press releases. Public Relations Review, 14(2): 45-49.

Morton, L. P. \& RAMSEY, S. (1994). A benchmark study of the PR news wire. Public Relations Review, 20(2): 171.

Morton, L. P. \& Warren, J. (1992). Acceptance characteristics of hometown press releases. Public Relations Review, 18(4): 385-390.

Sanford, A. J., Moar, K. \& Garrod, S. C. (1988). Proper names as controllers of discourse focus. Language and Speech, 31: 43-56.

Stocking, S. H. (1985). Effect of public relations efforts on media visibility of organizations" en Journalism Quarterly, 62(2): 358-366.

Stone, G. C. \& Morrison, J. (1976). Content as a key to the purpose of community newspapers. Journalism Quarterly, 53: 494-498. 
Tuchman, G. (1978). Making news: a study in the construction of reality. Nueva York: Free Press.

Walters, L. M. \& Walters, T. N. (1992). Environment of confidence: daily news paper use of press releases. Public Relations Review, 18: 31-46.

Whitlow, S. S. (1977). How male and female gatekeepers respond to news stories of women. Journalism Quarterly, 54 (3): 573-579.

Wilkie, T. (1996). Sources in science: who can we trust?. The Lancet, 347: 1308-1311.

\section{Lorena Busto-Salinas}

Licenciada en Comunicación Audiovisual por la Universidad de Burgos (UBU) en 2008 con premio extraordinario. Es becaria FPI de la misma universidad, donde ha presentado su DEA sobre comunicación institucional y muy en especial en torno a la nota de prensa. Forma parte del grupo de investigación Comunicación, Difusión y Publicidad de la Cultura y del Patrimonio de la Universidad de Burgos (CAYPAT). Ha participado en varios contratos de $\mathrm{I}+\mathrm{D}+\mathrm{i}$ sobre la divulgación del patrimonio y ha contribuido en la redacción de los libros Un siglo de fotografía en Burgos. 1840-1940 y El Patrimonio Histórico de Castilla y León. Tradición y Cultura. 